\title{
Discussion on the development trend of agricultural science and Technology Advisory Service Based on Artificial Intelligence
}

\author{
Jun $\mathrm{Yu}^{1,2}$, Qingfeng Wei ${ }^{1,2}$, Changshou Luo ${ }^{1,2, *}$, Qingfeng Wei ${ }^{1,2}$ \\ ${ }^{1}$ Institute of Agricultural Information and Economics. Beijing Academy of Agriculture and Forestry \\ Sciences, Beijing, China \\ ${ }^{2}$ Beijing Research Center of Engineering Technology on Rural Distance Information Service, Beijing, \\ China
}

*Corresponding author e-mail: Luocs@agri.ac.cn

Keywords: Agricultural science and Technology, Advisory Service, Artificial Intelligence.

\begin{abstract}
The development of artificial intelligence is the future direction of agricultural science and technology consulting services. This paper discusses the development of agricultural science and technology consulting service of artificial intelligence need, developing character, facing constraints and recommendations designed to provide advisory services on agricultural science and technology of artificial intelligence implementation and provides references for further promotion.
\end{abstract}

\section{Introduction}

At present, artificial consulting solutions service has been unable to fully meet the user requirement in an era of "Internet + smart devices". Urgent need to improve service capabilities to meet the need for convenient, personalized and accurate service. The combination of agricultural science and technology consulting service based on artificial intelligence and expert telephone consultation service, is the appropriate way for upgrading agricultural science and technology consulting service, also an important direction for the development of agricultural science and technology consulting services in the future. This paper mainly discusses the development of artificial intelligence (AI in short) in agricultural science and technology consultation.

\section{The advantage of application AI in agricultural science and technology consultation}

\subsection{Reduce service costs and improve service efficiency}

The use of artificial intelligence to carry out agricultural science and technology advisory services mainly to take the "knowledge + artificial intelligence + Internet" approach. It can make full use of expert intelligence resources, effectively alleviate the contradiction between the limited agricultural experts and a large number of industrial technology support needs. And can provide all day, efficient operation of the advisory services. Take the hotline of Beijin 12396 for example, along with the increase in the number of advisory, it leads to long time wait, reply miss, and personnel costs increase. Those problems has became a abstacle to service lever develope. It urgents need to the artificial intelligence solutions.

\subsection{Enhance the scientific nature of services}

Agricultural science and technology advisory service based on artificial intelligence mainly use the agricultural large data base to solve the agriculture problem. Therefore, it is more scientific and rigorous.

\subsection{Promote the development of service standardization}

The operation of agricultural science and technology consulting service based on AI will establish a set of data generating, application and consulting service mode which are in line with the intelligent demand. It needs to be carried out on the basis of uniform standards of data collection, data processing, data sharing, and service access, etc. These will support the long-term development and application of agricultural science and technology consulting services based on AI. 


\subsection{Promote the service accuracy}

AI application will rescue the service staff and experts from the complex affairs, so that they have the vigour to research the service object, the market demand and realize the service precision, the multiplication.

\section{The characteristic of the agricultural science and technology advisory service system based on AI}

The research of AI in agricultural science and technology consulting service is a systematic process, which includes agricultural ontology, natural language understanding, automatic programming, expert system, neural network, autonomous learning and so on. It is based on the agricultural ontology, constructs knowledge objects, and study the acquisition way of agricultural knowledge. It main features includes:

\subsection{Agricultural ontology is the foundation of construction}

Agricultural knowledge ontology is a linear structure, which is divided into layers according to classification. At the same time, based on the classification object, the dependency pattern is constructed such as classification and attribute, object and instance. Through the ontology to achieve artificial intelligence, automatic induction.

\subsection{Agricultural science and technology data is guarantee.}

Question and answer is the basis for the establishment of AI system of agricultural science and technology consulting services. Through agricultural science and technology data, a large number of question and answers can be sorted out.

\subsection{The system has the ability to inspire, learn and deduce}

Heuristic learning and logical reasoning is the basic characteristics of agricultural science and technology consulting service based on AI. It is based on the specific knowledge, and deals with knowledge using human thinking.

\subsection{Need the support of agricultural science and technology services cloud}

Agricultural science and technology data in the use of artificial intelligence, the demand for computing power is far beyond the computing power of their own IT architecture, then you need to constantly increase the system hardware input to achieve the scalability of the system. In addition, due to the limitations of the traditional parallel programming model, it needs a new parallel programming which is easy to learn, use and deploy. Agricultural science and technology service cloud are a good solution to this problem.

\section{Difficulties of constructing agricultural science and technology consulting service system based on AI}

The research of AI includes knowledge engineering, natural language understanding, computer graphics technology. Compared with other industries, the biggest feature is the huge complexity of agricultural knowledge system, and the constraints of user's ability to accept. Therefore, the intelligent construction is relatively complex. The main difficulties are summarized as follows:

\subsection{Large and complex agricultural knowledge system construction}

The construction of the ontology and semantic web in agricultural science and technology consulting service based on AI is a long process. Semantic network construction based on agricultural ontology. The imperfection of the agricultural ontology construction has also restricted the construction of the semantic network of agricultural consulting service.

\subsection{The establishment of answers and questions pairs}

Agricultural issues involves a wide range of large. The answer to the question requires expert support. It is difficult to form such an effective and scientific question and answer pairs.

\subsection{Precision of AI semantic understanding and recognition}

Agricultural terminology is not standard, and also contains accent dialect. It is the main challenges for the understanding and recognition of natural language. 


\section{Development suggessions}

Agricultural ontology construction is difficult. It should be based on the existing agricultural knowledge base, to construct the agricultural ontology which is suitable for the current service demand. In the design of ontology, the scalability should be fully considered at the same time.

Establishment and extraction of semantic web. At present, the extraction of semantic web is not mature. A feasible scheme is to use the semantic segmentation system of ICTCLAS to realize the extraction of words, to construct the semantic analysis network, to complete the matching of elements, and achieve the fuzzy semantic matching.

The pairs construction of questions and answers. The establishment of questions and answers pairs should be based on ontology and according to the standard. For example, take tomato as the agricultural ontology object, and then around the tomato to build all the pairs. When the user consults tomato problems, always can find the answer.

The agricultural science and technology consulting service based on AI is different from the automatic question and answer in the open field. It requires that the questions and answers must be scientific, practical and professional. Take the question of "what kind of tomato to choose? " for example: Cannot feedback the tomato seed information to the user, but also should consider the cultivation of regional, planting environment and other factors to give the correct answer. Therefore, solve the problem of consultation, a perfect knowledge system is needed.

\section{Conclusion}

Agricultural science and technology advisory services based on AI is an inevitable result of the "Internet plus" based on age. The research of agricultural science and technology consulting service based on AI will improve the level of agricultural science and technology service, and also promote the development of service industry. However, its development is limited by the factors such as agricultural knowledge ontology, computer technology, neural network algorithm and user's quality. Therefore, the research in this field is a long process. In this process, the level of agricultural science and technology advisory services based on AI will be higher and higher.

Carry out the training work, should make full use of information technology means, to reduce the training cost, expand training radiating surface, and provide training opportunities to more people.

\section{Acknowledgments}

The research work was supported by the fund for agriculture of Beijing municipal commission of rural affai: The application of APP and "zhixuntong" products in the management and service of general agricultural technicians, the young scientist fund of BAAFS: Research on agricultural scientific technical online counseling system based on the knowledge map (NO.QNJJ201534), the innovation fund of BAAFS: Agricultural water-saving technology training and counseling service demonstration project (NO.KJCX20151409).

\section{References}

[1] Yuxiang Shi. Modern agricultural information technology and agricultural information consulting service. Jiangsu rural economy. 2011(5):68-69.

[2] Qing Yang. From "Internet +" to "+Artificial intelligence". Journal of Chongqing University of Technology. 2015,29(10):4-4

[3] Shaobo Yu, Shoulun Hu. Artificial intelligence and neural network: VIP information. 1990 (2): 64-68. 